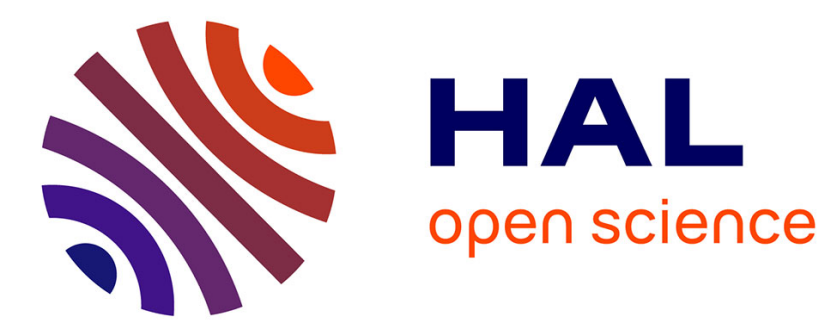

\title{
Optical characterization of photosensitive AMTIR-1 chalcogenide thin layers deposited by electron beam deposition
}

Alexandre Joerg, Fabien Lemarchand, Mengxue Zhang, Michel Lequime, Julien Lumeau

\section{To cite this version:}

Alexandre Joerg, Fabien Lemarchand, Mengxue Zhang, Michel Lequime, Julien Lumeau. Optical characterization of photosensitive AMTIR-1 chalcogenide thin layers deposited by electron beam deposition. Journal of Non-Crystalline Solids, 2016, 442, pp.22-28. 10.1016/j.jnoncrysol.2016.03.018 . hal-01300747

\section{HAL Id: hal-01300747 \\ https://hal.science/hal-01300747}

Submitted on 13 Mar 2019

HAL is a multi-disciplinary open access archive for the deposit and dissemination of scientific research documents, whether they are published or not. The documents may come from teaching and research institutions in France or abroad, or from public or private research centers.
L'archive ouverte pluridisciplinaire HAL, est destinée au dépôt et à la diffusion de documents scientifiques de niveau recherche, publiés ou non, émanant des établissements d'enseignement et de recherche français ou étrangers, des laboratoires publics ou privés. 


\title{
Optical characterization of photosensitive AMTIR-1 chalcogenide thin layers deposited by electron beam deposition
}

Alexandre Joerg ${ }^{1}$, Fabien Lemarchand ${ }^{1}$, Mengxue Zhang $^{2}$, Michel Lequime ${ }^{1}$ and Julien Lumeau $^{1}$

${ }^{1}$ Aix Marseille Université, CNRS, Centrale Marseille, Institut Fresnel UMR 7249, 13013 Marseille, France

${ }^{2}$ Chimie ParisTech, PSL Research University, CNRS, Institut de Recherche de Chimie Paris (IRCP), F - 75005 Paris, France

\begin{abstract}
:
Amorphous $\mathrm{Ge}_{33} \mathrm{As}_{12} \mathrm{Se}_{55}$ films have been deposited by electron beam physical vapor deposition and their optical properties have been studied using reverse engineering on spectrophotometric measurements. Definition of the different optical constants were made by Tauc-Lorentz model allowing simultaneous characterization in high and low absorption area. Moreover an investigation of the layer's photosensitivity with an exposure wavelength at $\sim 808 \mathrm{~nm}$ has been carried out and reveals a photo-bleaching effect generating up to 0.04 refractive index variation at $1 \mu \mathrm{m}$. Finally the stability of the fabricated layers is studied.
\end{abstract}

\section{Keywords:}

Chalcogenide glass, optical coating, photosensitivity, spectrophotometry 


\section{Introduction}

Chalcogenide glasses are now commonly used in different applications such as solar cells, infrared sensors [1] or phase-change memories [2,3]. These glasses consists of chalcogens elements from the sixteenth column of the periodic table (excepted oxygen) covalently bonded to heavy elements such as: As, Ge, Sb, Ga, Te, or Si. Due to their weak inter-atomic bonds, chalcogenide glasses are transparent in the near and the mid-infrared regions of spectrum. For example the sulfides transmit up to $11 \mu \mathrm{m}$, selenides up to $15 \mu \mathrm{m}$ and tellurides up to $20 \mu \mathrm{m}$. In addition, chalcogenide glasses were already deposited in thin films to fabricate optoelectronic components [4-6] and ultra-narrow bandpass filters [7]. A striking propertiy of chalcogenide glasses is their photosensitivity $[8,9]$ i.e. the modification of the chemical bonds under an exposure light with wavelength included in the bandgap of the material. Actually, various photo-induced mechanisms were observed in chalcogenide layers such as photoionization [10], photoexpansion [11,12], photodensification [13], photocrystallization [14], amorphisation [15], photodarkening [16,17] or photobleaching [18]. These effects have been widely studied to create active optical interference filers [19,20] or to write Bragg grating in planar waveguides [21-24]

In this work we have investigated the deposition of $\mathrm{Ge}_{33} \mathrm{As}_{12} \mathrm{Se}_{55}$ thin films. This glass has been selected due to its commercial availability and known photosensitivity. Some previous works have already shown that it can be deposited into thin films using by pulsed laser deposition [2527] or thermal evaporation [28-30] or electron beam deposition (EBD) [31], because it allows producing homogeneous thick and large aperture films that will be compatible with the production of multilayer optical interference filters. Using reverse engineering coupled with a Tauc Lorentz model, the optical properties of single layers were determined and a precise study of the photosensitive effects is presented. Finally a systematic study of the stability of the 
fabricated films is performed, showing that these layers are compatible with the production of complex optical elements.

\section{Experiments}

Chalcogenide glasses with composition $\mathrm{Ge}_{33} \mathrm{As}_{12} \mathrm{Se}_{55}$ and commonly known as AMTIR-1 were used in this study. These glasses were produced by the Amorphous Material Company. Cylindrical samples with $25 \mathrm{~mm}$ diameter and thickness of about $12 \mathrm{~mm}$ were placed on a Mo liner inside a BALZERS BAK600 system with residual pressure within the chamber of $\sim 10^{-6} \mathrm{mBar}$. Thin single-layers were fabricated by EBD on fused silica substrates which were cleaned in an ultrasonic bath. Various films with thicknesses ranging between 300 and $700 \mathrm{~nm}$ were fabricated. AMTIR-1 having a low glass transition and melting temperatures (e.g. $\mathrm{T}_{\mathrm{g}}=$ $362^{\circ} \mathrm{C}$ ), very low and stable deposition rate could not be achieved. The deposition rate was thus set to $10 \mathrm{~A} / \mathrm{s}$ and was controlled by an Inficon quartz crystal monitor XTC/2. This speed allowed securing stable deposition speed with fluctuations not exceeding $\pm 20 \%$ after optimizing the PID parameters.

Transmission and reflection spectra of each single-layer were measured with a Perkin Elmer Lambda 1050 spectrophotometer in the spectral range from 600 to $1800 \mathrm{~nm}$ at an angle of incidence of $8^{\circ}$. A special module from OMT solutions was used to perform this measurement. This module allows accurate and absolute measurement of both transmission and reflection spectra of a sample, without moving or replacing the sample and without reflection etalons as transmission baseline is used to both calibrate the transmission and reflection spectra. Such a system is crucial for obtaining high accuracy measurements and determinations of the optical properties of the layers. Typical precision of the measured transmission and reflection intensity

is no larger than $0.1 \%$. Composition of the thin films compare to the bulk material were also studied using Energy-dispersive X-ray spectroscopy (EDS) 
AMTIR-1 being a photosensitive material, kinetics of refractive index change on dosage of actinic radiation were studied. Radiation from a $200 \mu \mathrm{m}$-fiber-coupled laser diode centered at $808 \mathrm{~nm}$, with a spectral bandwidth of $2 \mathrm{~nm}$ and a beam diameter of $10 \mathrm{~mm}$ was used to perform exposure. Power density in place of the sample was $15 \mathrm{~W} / \mathrm{cm}^{2}$. Samples were exposed with dosages up to $1.1 \times 10^{2} \mathrm{~kJ} / \mathrm{cm}^{2}$. Between exposures, samples were kept into dark boxes in order to secure that no parasitic exposure from external source would modify the layers properties. After each exposure, reflection and transmission spectra were measured using the procedure that was described above. Customs algorithms were finally developed in order to extract the optical parameters of the single layers: the refractive index dispersion $n(\lambda)$, the extinction coefficient dispersion $\mathrm{k}(\lambda)$, the thickness $\mathrm{t}$, and the band gap E0.

\section{Determination of AMTIR-1 single layer optical constants}

\section{a. Tauc-Lorenz Model}

We used reflection and transmission spectra measured on AMTIR-1 single layers to determine their optical constants. Typical spectra measured on a $500 \mu \mathrm{m}$ thick layer is presented in Figure 1. One can see two distinct regions, one above $700 \mathrm{~nm}$ that presents oscillations of the signal, typical of the interferences occurring within the layer, and a second region below $700 \mathrm{~nm}$, where the layer presents high absorption. Optical constants of thin film materials, are generally determined using the Swanepoel method [32]. This method involves determining the envelopes $\mathrm{T}_{\mathrm{M}}$ and $\mathrm{T}_{\mathrm{m}}$ describing respectively the spectral dependence of the amplitude of the maxima and the minima of the measured interference pattern. This method provides with a simple method for evaluating the constants of single layers in low absorbing regions i.e. where $\mathrm{k}<<0.01$. However, as illustrated on Fig. 1, AMTIR-1 thin films have large absorption in the spectral regions below $900 \mathrm{~nm}$ due to intrinsic absorption of the glass matrix. In addition, this study is intended to provide an accurate determination of the optical properties of these layers that will 
be further used for the design of complex multilayer filters. To achieve, the Tauc-Lorentz (TL) [33-36] method was implemented for modeling the dispersion of optical constants. It is important to stress that this method was shown to be an accurate method for modeling interband absorption [37] and high absorbing optical coating materials [38], e.g. silicon ( $\mathrm{Si}$ ) [39,40], hydrogenate silicon (a-Si:H) [41]...

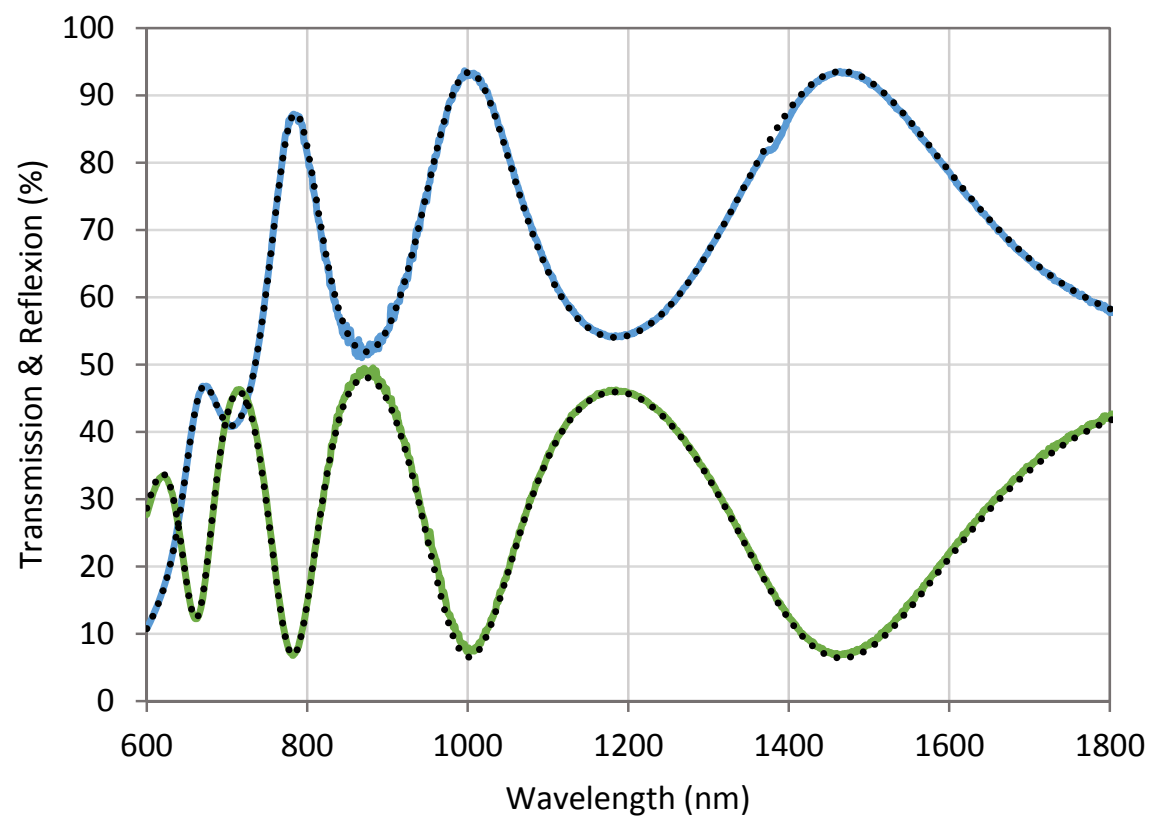

Figure 1: Measured and theoretical (resulting from TL fitting) spectral dependences of the transmission and reflection of an AMTIR-1 single layer

TL optical model is derived from Kramers-Kronig [42] expression where electric permittivity can be express as:

$$
\varepsilon(E)=\varepsilon_{1}(E)+i \varepsilon_{2}(E)
$$

And $\mathrm{N}$ the complex refractive index is linked with electric permittivity by the following formula:

$$
\varepsilon(E)=N(E)^{2}=(n(E)+i k(E))^{2}
$$

Real and imaginary part of electric permittivity $\varepsilon_{1}(E)$ and $\varepsilon_{2}(E)$ are given by: 


$$
\begin{aligned}
& \varepsilon_{2}(E)=\left\{\begin{array}{c}
\sum_{i=1}^{q} \frac{A_{i} E_{0 i} C_{i}\left(E-E_{T L}\right)^{2}}{\left(E^{2}-E_{0 i}^{2}\right)^{2}+C_{i}{ }^{2} E^{2}} \frac{1}{E}, E>E_{T L} \\
0, E \leq E_{T L}
\end{array}\right. \\
& \varepsilon_{1}(E)=\varepsilon_{\infty}+\sum_{i=1}^{q}-A_{i} E_{0 i} C_{i} \frac{E^{2}+E_{T L}^{2}}{\pi \zeta_{4 i} E} \ln \left(\frac{\left|E_{T L}-E\right|}{E_{T L}+E}\right) \\
&+\frac{2 A_{i} E_{0 i} C_{i} E_{T L}}{\pi \zeta_{4 i}} \ln \left(\frac{\left|E_{T L}-E\right|\left(E_{T L}+E\right)}{\sqrt{\left(E_{0 i}^{2}-E_{T L}^{2}\right)^{2}}+C_{i}{ }^{2} E_{T L}^{2}}\right) \\
&+\frac{A_{i} C_{i} a_{L i}}{2 \pi \zeta_{4 i} \alpha_{i} E_{0 i}} \ln \left(\frac{E_{0 i}^{2}+E_{T L}^{2}+\alpha_{i} E_{T L}}{E_{0 i}^{2}+E_{T L}^{2}-\alpha_{i} E_{T L}}\right) \\
&-\frac{A_{i} \alpha_{A i}}{\pi \zeta_{4} E_{0}}\left[\pi-\arctan \frac{2 E_{T L}+\alpha_{i}}{C_{i}}-\arctan \frac{2 E_{T L}-\alpha_{i}}{C_{i}}\right] \\
&+4 A_{i} E_{0 i} E_{T L} \frac{E^{2}-\gamma_{i}{ }^{2}}{\pi \zeta_{4 i} \alpha_{i}}\left[\frac{\pi}{2}-\arctan \frac{2\left(E_{T L}^{2}-\gamma_{i}^{2}\right)}{\alpha_{i} C_{i}}\right]
\end{aligned}
$$

Where:

$$
\begin{aligned}
& a_{L i}=\left(E_{T L}^{2}-E_{0 i}^{2}\right) E^{2}+E_{T L}^{2} C_{i}^{2}-E_{0 i}^{2}\left(E_{0 i}^{2}+3 E_{T L}^{2}\right) \\
& \alpha_{A i}=E^{2}-E_{0 i}^{2}\left(E_{0 i}^{2}+E_{T L}^{2}\right)+E_{T L}^{2} C_{i}^{2} \\
& \gamma_{i}=\left(E_{0 i}^{2}-\frac{C_{i}^{2}}{2}\right)^{1 / 2} \\
& \alpha_{i}=\left(4 E_{0 i}^{2}-C_{i}^{2}\right)^{1 / 2} \\
& \zeta_{4 i}=\left(E^{2}-E_{0 i}^{2}\right)^{2}+C_{i}^{2} E^{2}
\end{aligned}
$$

In case of AMTIR-1 single layers, the parameters of interest are: ETL, the band gap energy of the materials, $\mathrm{E}_{0}$, the central peak of energy, $\mathrm{C}$ the broadening parameter, A the amplitude, $\varepsilon_{\infty}$ the high frequency dielectric constant and the thickness. The total number of parameters to 
determine is equal to $\mathrm{p}=3\left(\mathrm{E}_{\mathrm{TL}}, \varepsilon_{\infty}, \mathrm{t}\right)+3 \mathrm{q}\left(\mathrm{A}_{\mathrm{i}}, \mathrm{C}_{\mathrm{i}}, \mathrm{E}_{0 \mathrm{i}}\right)$ where $\mathrm{q}$ is the number of oscillators used in Tauc-Lorentz formalism. Generally, the q parameter is equal to one, e.g. for $\mathrm{Ta}_{2} \mathrm{O}_{5}$ [39], $\mathrm{HfO}_{2}$ [43], but sometimes, increasing the number of oscillators is required in order to get an optimal estimation of optical functions, e.g. $\mathrm{Ag}_{2} \mathrm{O}$ [44]. However, one must keep in mind that like in any other mathematical decomposition of a physical curve, the number of free parameters must be kept to minimum in order to provide an error function between model and experimental curve equal to the measurement precision.

Optimization of the model was performed using Matlab and more precisely the programmed "fmincon" function. This algorithm allows for searching the minimum of constrained functions. As it's an optimization program, a converging error function (EF) needs to be defined. A pondered sum of the quadratic errors of the fitted transmission and reflection was chosen:

$$
E F=\sqrt{\frac{1}{2 N} \sum_{j=1}^{N} \alpha\left(R_{\text {cal } j}(X, t)-R_{\text {spec } j}\right)^{2}+(1-\alpha)\left(T_{\text {cal } j}(X, t)-T_{\text {spec } j}\right)^{2}}
$$

where $\mathrm{X}$ is a vector of all the derived parameter, $\mathrm{t}$ the thickness, $\mathrm{R}_{\mathrm{spec}}$ and $\mathrm{T}_{\mathrm{spec}}$, the reflection and transmission spectra measured with the spectrophotometer, $\mathrm{R}_{\mathrm{cal}}$ and $\mathrm{T}_{\mathrm{cal}}$ are the calculated reflection and transmission spectra. $\alpha$, which is a coefficient between 0 and 1 that has to be assessed, was fixed at 0.5 in this study in order to place the same weight on transmission and reflection spectra and therefore to allow for accurate fitting of both spectra.

\section{b. Results: Optical constants determination}

The transmittance (Tcal) and reflectance (Rcal) curves were modeled with the TL formalism using one $(\mathrm{q}=1)$ or two oscillators $(\mathrm{q}=2)$. Table 1 summarizes the fitting parameters required in both cases. One can see that with only one oscillator, the RMS error of the transmission curve is twice larger and the EF is three times larger than with the 2-oscillator model. Therefore, this 
result shows that a two-oscillator model is required for an accurate description of the experimental curves (Figure 1). Regarding the values, one can see that the bandgap energy that were extracted tend to be lower than the one reported in the literature $[29,30]$. This result is however not really surprising as it is well known that the parameters of the coatings highly depend on the deposition technique and parameters.

Based on this model, we plotted the corresponding refractive index and extinction coefficient dispersion curves in Figure 2. Based on this determination, the deposited AMTIR-1 thin film is $630 \mathrm{~nm}$ thick. Its refractive index is higher than 2.9 in the visible range while it decreases below 2.7 in near infrared region. Regarding absorption this one becomes negligible, i.e. below 0.001 above $875 \mathrm{~nm}$ while it severely increases below $750 \mathrm{~nm}$ due to the intrinsic absorption of the glass matrix. This result means that applications of AMTIR-1 lie in the $1+$ micron region. These data are slightly higher than the one reported in the literature [29] and more than 0.1 higher than the refractive index of bulk AMTIR-1 glass, confirming that the material has been affected by the deposition process, producing a non-photo-stable material.

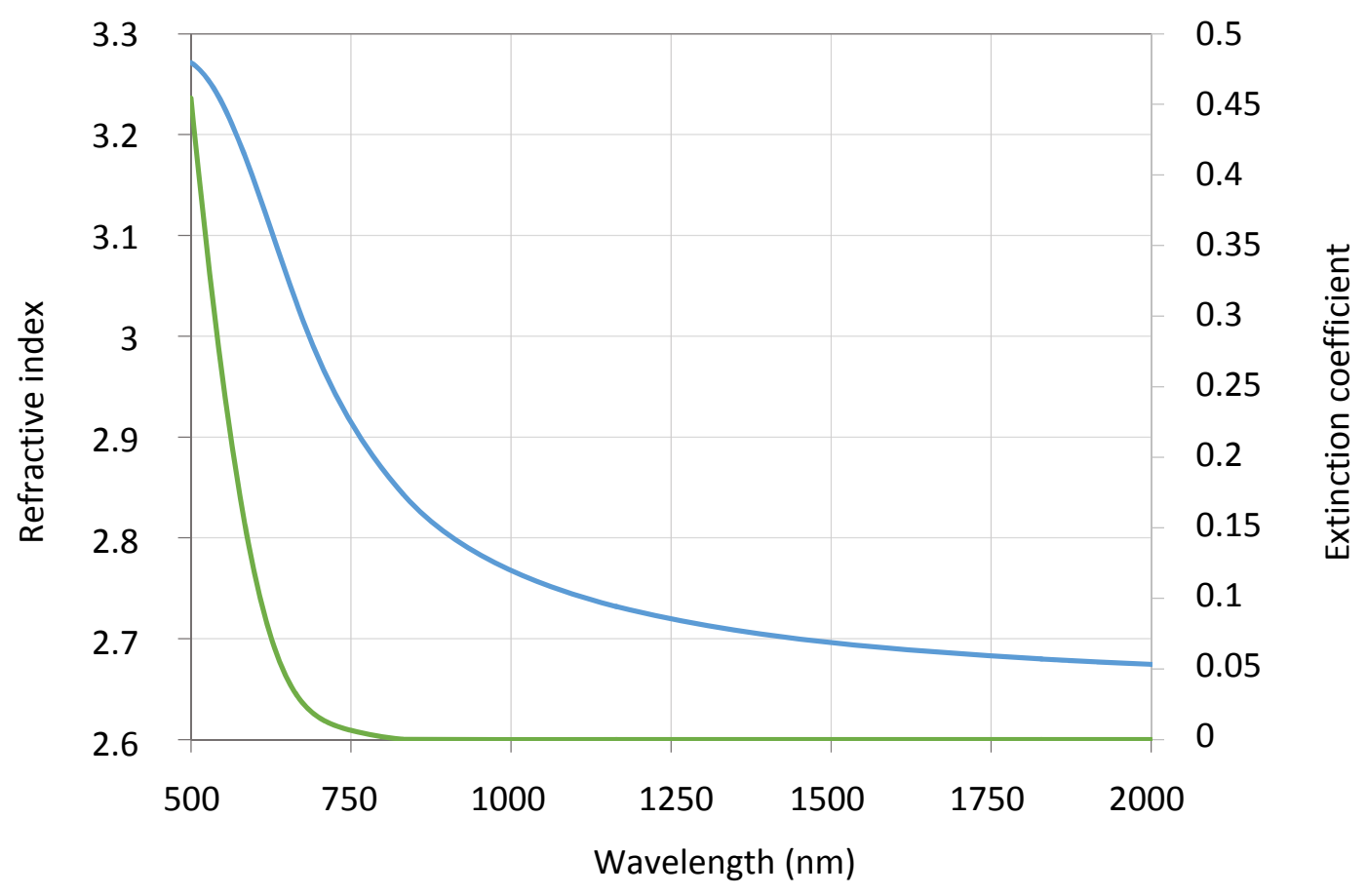


Figure 2: Refractive index (blue curve) and extinction coefficient (green curve) dispersion of AMTIR-1 layers

Table 1. Parameters used for TL fitting of AMTIR-1 spectroscopic data using 1 and 2 oscillators. Due to the level of noise of our measurements, the precision for each of the determined parameters is better than $1 \%$.

\begin{tabular}{ccccccccccc}
\hline & $\boldsymbol{E}$ & $\mathbf{E T L}$ & $\mathbf{A 1}$ & $\mathbf{E 0 1}$ & $\mathbf{C 1}$ & $\mathbf{A 2}$ & $\mathbf{E 0 2}$ & $\mathbf{C 2}$ & $\mathbf{t}$ & $\mathbf{E F}$ \\
\hline $\mathbf{q = 1}$ & 1.76 & 1,49 & 80,43 & 4,13 & 3,33 & & none & & 549.1 & 0.0080 \\
$\mathbf{q = 2}$ & 1.82 & 1.46 & 87.47 & 3.91 & 3.96 & 4.92 & -1.80 & 0.71 & 548.1 & 0.0040 \\
\hline
\end{tabular}

Actually, the previously presented data are of high importance when it comes to AMTIR-1 photosensitivity. In fact to generate any photosensitive effect, the layer needs to be exposed to actinic radiation with wavelength within the absorbing region of the glass. It is therefore of prime interest to precisely know how to describe the dispersion curve of the extinction coefficient and to estimate the band gap. This is directly obtained with the TL model. Actually, the wavelength of exposure must be chosen depending on the application: the higher the absorption at the wavelength of exposure, the faster the refractive index change, however, the higher the gradient of induced refractive index change through the layer depth [45]. Depending on the application, i.e. whether a large [46] or a low [47], it is possible to determine the best exposure wavelength that will allow rapidly inducing a large refractive index change with minimal grating of index. In the case of this study, we used a laser diode centered at $808 \mathrm{~nm}$. Such laser sources combine high energy and good beam quality that make them suitable for fast and structured exposure systems [46].

\section{c. Study of the speed rate deposition}


In order to have stable and repeatable refractive index between each deposition as well to assess the possible gradient of index that could be produced the layer fabrication associated with deposition speed fluctuations, that effect of the rate deposition on AMTIR-1 refractive index was studied. 3 layers with identical thickness of $\sim 350 \mathrm{~nm}$ were deposited with 3 different rates: 5, 10 and $20 \mathrm{~A} / \mathrm{s}$. Then the optical constants were extracted with the previous described method. To compare the results with another classical material, the same work was done on $150 \mathrm{~nm}$ $\mathrm{Ta}_{2} \mathrm{O}_{5}$ thick layer. The $\mathrm{Ta}_{2} \mathrm{O}_{5}$ deposition rates were fixed to classical values, i.e. 2,3 and $6 \mathrm{~A} / \mathrm{s}$ (Figures 3 and 4).

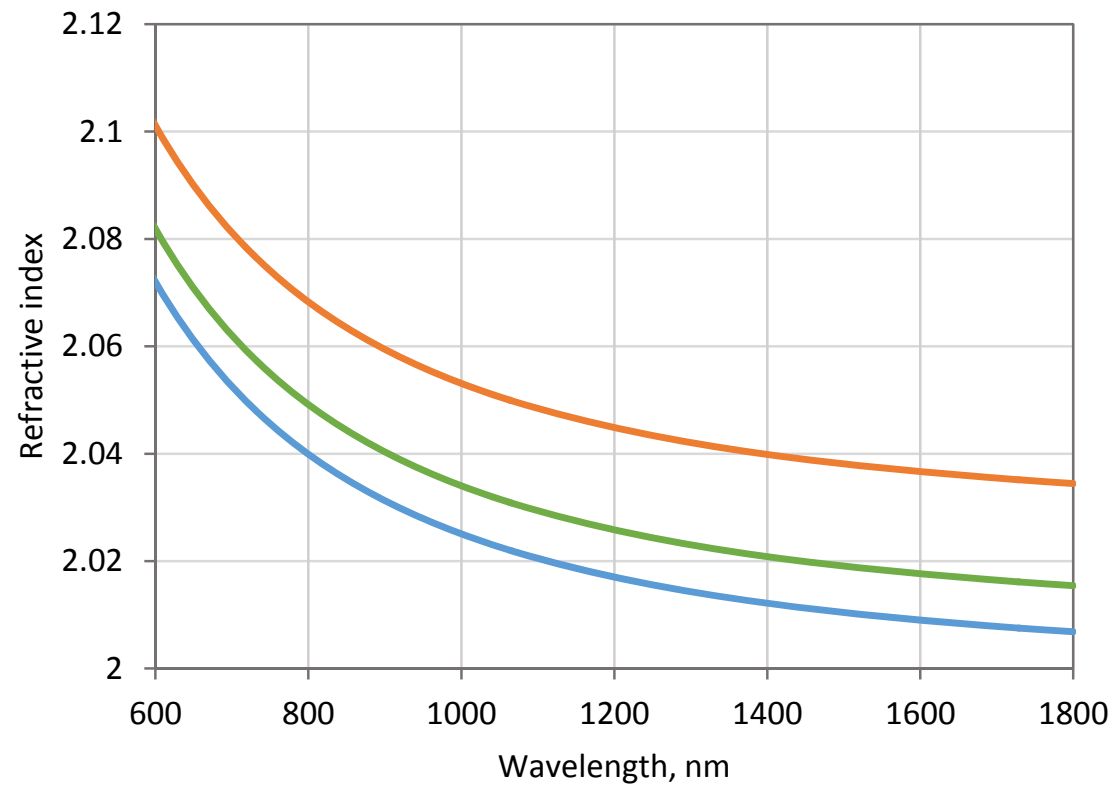

Figure 3: $\mathrm{Ta}_{2} \mathrm{O}_{5}$ refractive index dispersion determined on sample fabricated with different deposition rates: in blue $-2 \mathrm{~A} / \mathrm{s}$, in green $3 \mathrm{~A} / \mathrm{s}$, in orange $6 \mathrm{~A} / \mathrm{s}$ 


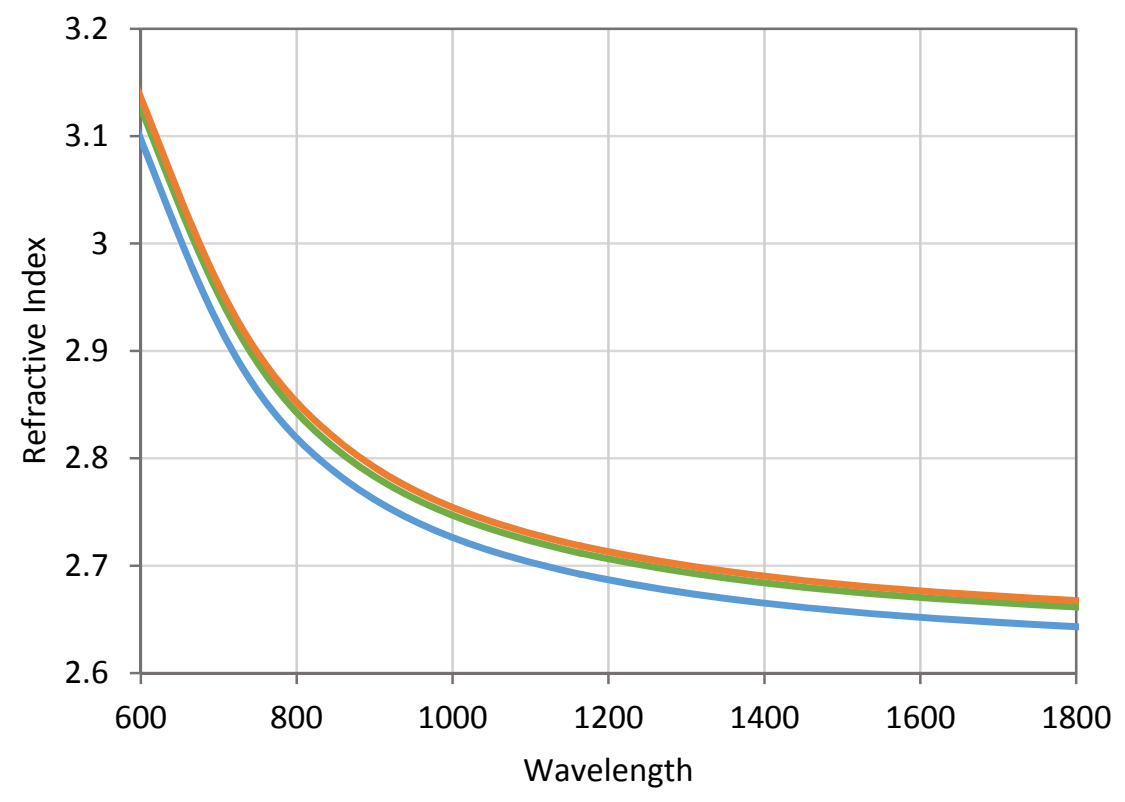

Figure 4: AMTIR-1 refractive index dispersion determined on sample fabricated with different deposition rates: in blue - 5A/s, in green $10 \mathrm{~A} / \mathrm{s}$, in orange $20 \mathrm{~A} /$

For the both materials, a higher deposition rate leads to a higher refractive index. But they have a different behaviors depending on the deposition rate. For example $\mathrm{Ta}_{2} \mathrm{O}_{5}$ layers seem to have a linearly increasing refractive index with deposition rate: 3 times higher deposition rate results in a 3 times higher refractive index increase for each wavelength. AMTIR-1 has a different behavior. A significant refractive index $(\Delta \mathrm{n}=0.02)$ variation is observed when deposition rate is changed from 5 to $10 \mathrm{~A} / \mathrm{s}$ and then barely changes $(\Delta \mathrm{n}<0.005)$ when it is further increased to $20 \mathrm{~A} / \mathrm{s}$. This result shows that for a high deposition rate, larger variation of deposition rate can be tolerated to obtain layers with uniform refractive index. For example for $1 \mu \mathrm{m}$ wavelength, to get an error on the refractive index not exceeding $5.10^{-3}$, at a deposition rate of $5 \mathrm{~A} / \mathrm{s}$ the variation should not exceed $0.5 \mathrm{~A} / \mathrm{s}$, but for $20 \mathrm{~A} / \mathrm{s}$, deviation up to $4 \mathrm{~A} / \mathrm{s}$ could be tolerated. Such fluctuations are compatible with the deposition technique that was implemented within this work. However, as already stated in Ref [30,48], one generally wants low deposition rates to produce film composition that is very close to the starting bulk materials. In our case, 
our goal was to produce optical quality, homogeneous and photosensitive layers, therefore higher deposition rate appear as optimal conditions for the first two parameters.

In addition, the composition of those layers has been investigated and determined by a nondestructive technique: Scanning Electron Microscopy with Energy Dispersive Analysis Systems (FESEM/EDS, Zeiss Gemini 1530). The EDS technique detects X-rays emitted from the sample during bombardment by an electron beam to characterize the elemental composition of the analyzed volume. The depth from where the X-rays originate depends on the material and the used primary electron energy. For the accelerating energy of $20 \mathrm{keV}$ used for our experiments, it is in the several hundreds of $\mathrm{nm}$ to $2 \mu \mathrm{m}$ range. Several measurements are performed on each sample. A Gaussian fit is performed to calculate the area under the peaks and then to calculate the weight or atomic percentage of each element. The results exposed on Figure 5 were obtained by averaging different local measurements on each substrate.

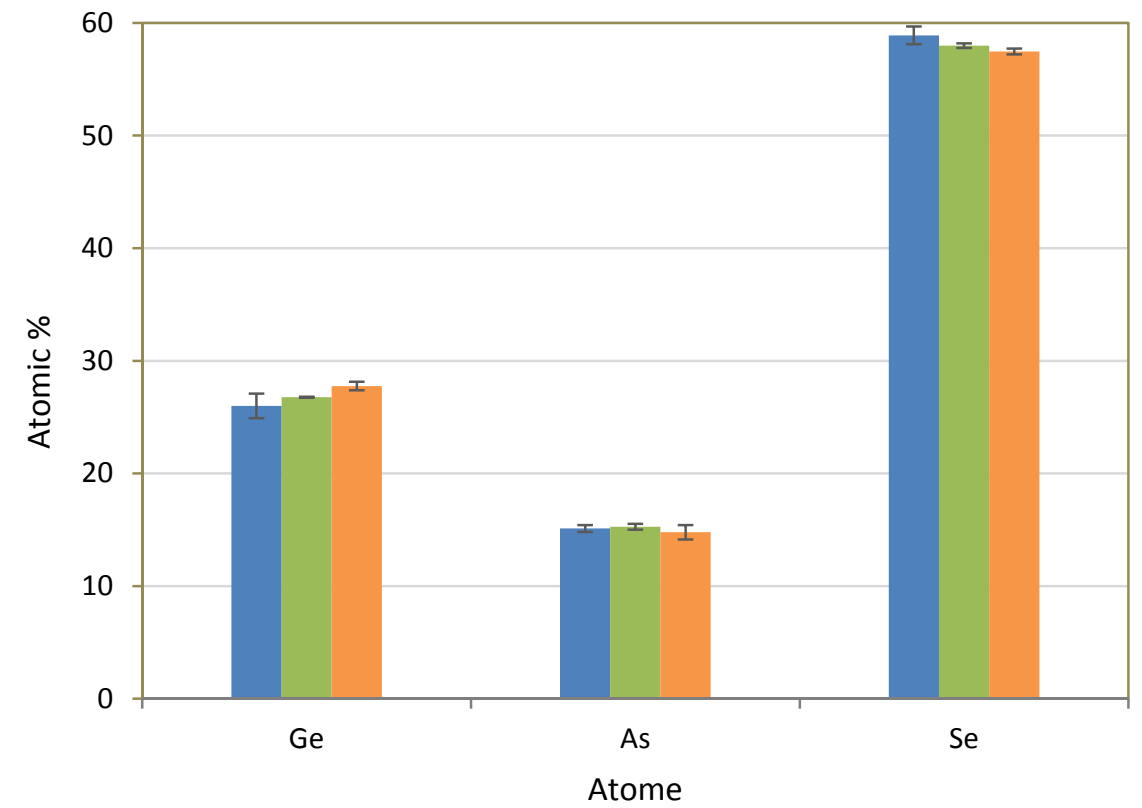

Figure 5: AMTIR-1 composition for different deposition rate: in blue - 5A/s, in green $10 \mathrm{~A} / \mathrm{s}$ and in orange $20 \mathrm{~A} / \mathrm{s}$ 
The precision reached is around $1 \%$ for each element. The composition measured on the layers significantly different to the one of the bulk materials, there is for example a $5.5 \%$ decrease of Ge concentration in comparison with original composition. Moreover, this result shows that within this range, the deposition rate has no significant impact on the final thin film composition. It also signifies that the variation of refractive index is not a result of the thin film composition change. This difference of refractive index is most likely a result of distinct layer densities associated with various kinetic energy during the evaporation process. Based on these results and in order to have a precise control on the deposited layer thicknesses, we choose to deposit the AMTR-1 layers at a rate of $10 \AA / \mathrm{s}$.

\section{Kinetics of photosensitivity in AMTIR-1 single layers}

\section{a. Photosensitivity}

The samples were exposed with diode laser centered at $808 \mathrm{~nm}$ for up to 48 hours. Figures 6 shows the typical transmission spectra before and after exposure (at saturation).

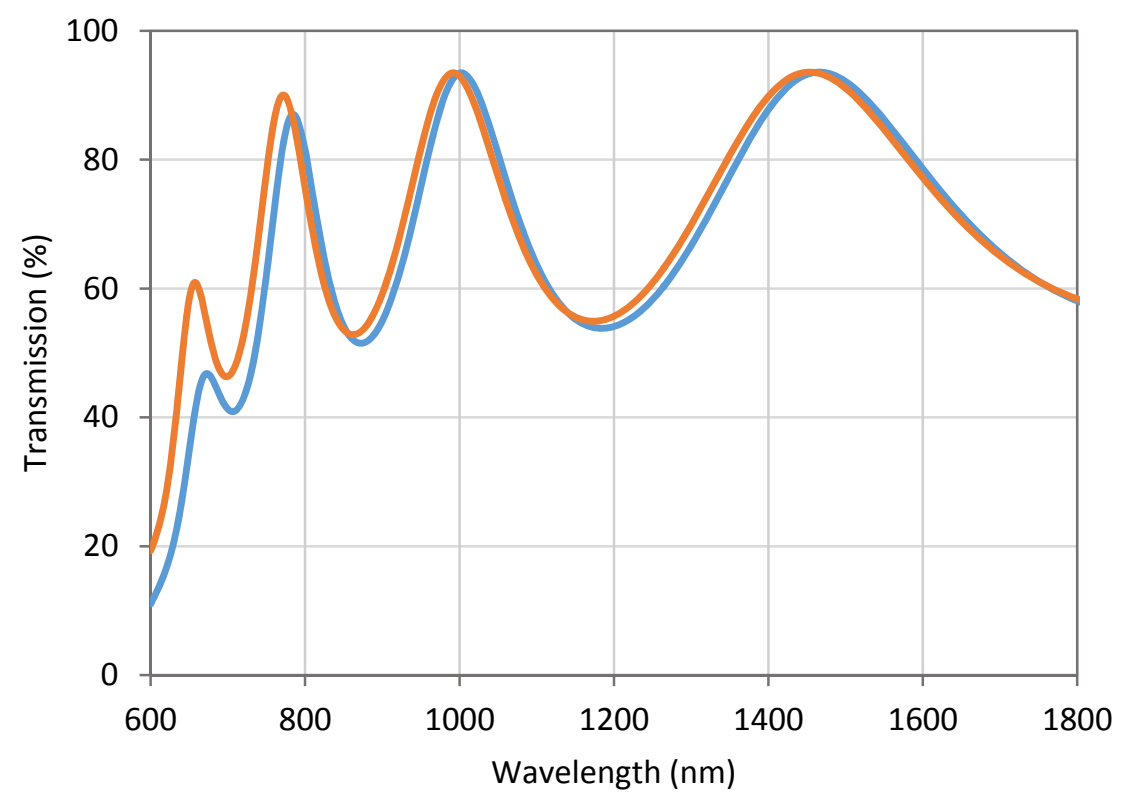


Figure 6: Spectral dependence of the transmission of AMTIR-1 single layers before and after exposure at $808 \mathrm{~nm}$ obtained by reverse engineering: in blue - transmission before exposure, in orange - transmission after exposure

First of all, one can see that after exposure, the transmission spectrum shifts towards shorter wavelength, meaning that optical thickness of the layer is decreasing. One can see that in nonabsorbing region (i.e. above $900 \mathrm{~nm}$ ), the amplitude of the maxima appears constant while the amplitude of the minima increases, resulting is a lower contrast of the interference fringes. This result illustrates that the refractive index of the layer is decreasing and therefore that at least part (if not all) of the optical thickness decrease is due to refractive index decrease. Finally, in higher absorbing region (i.e. around 700-800 nm), amplitude of the maxima increases after exposure, showing that absorption decreases within this region and therefore that bandgap structure is evolving. It is important to stress that these effects are large amplitude effects as they are easily seen without deep analysis and they also confirm a photo-bleaching effects. In order to quantify the photo-bleaching effects, we applied the TL formalisms to spectra measured after exposure. Parameters extracted from the TL fit are shown in Table 2 and corresponding spectral dependence of the refractive index and the extinction coefficient before and after exposure are shown in Fig. 7 and 8. One can see that the main effect of exposure at $808 \mathrm{~nm}$ is an overall decrease of the refractive index and a shift of the extinction coefficient towards lower wavelength. From these data, changes of the real and imaginary part of the refractive index at $800 \mathrm{~nm}$ can be extracted:

- $\Delta \mathrm{n}=\mathrm{n}_{\text {exposed }}-\mathrm{n}_{\text {unexposed }}=-0.05$

- $\Delta \mathrm{k}=$ kexposed-Kunexposed $=-0.002$

Those data, particularly the one of the refractive index change are comparable to the one reported in [29] and [25]. The imaginary part of the refractive index (k) decreases during the 
AMTIR-1 exposure. This decrease is due to an increase of the bandgap (ETL) that can be quantified using the TL model, from $1.46 \mathrm{eV}$ (before exposure) up to $1.48 \mathrm{eV}$.

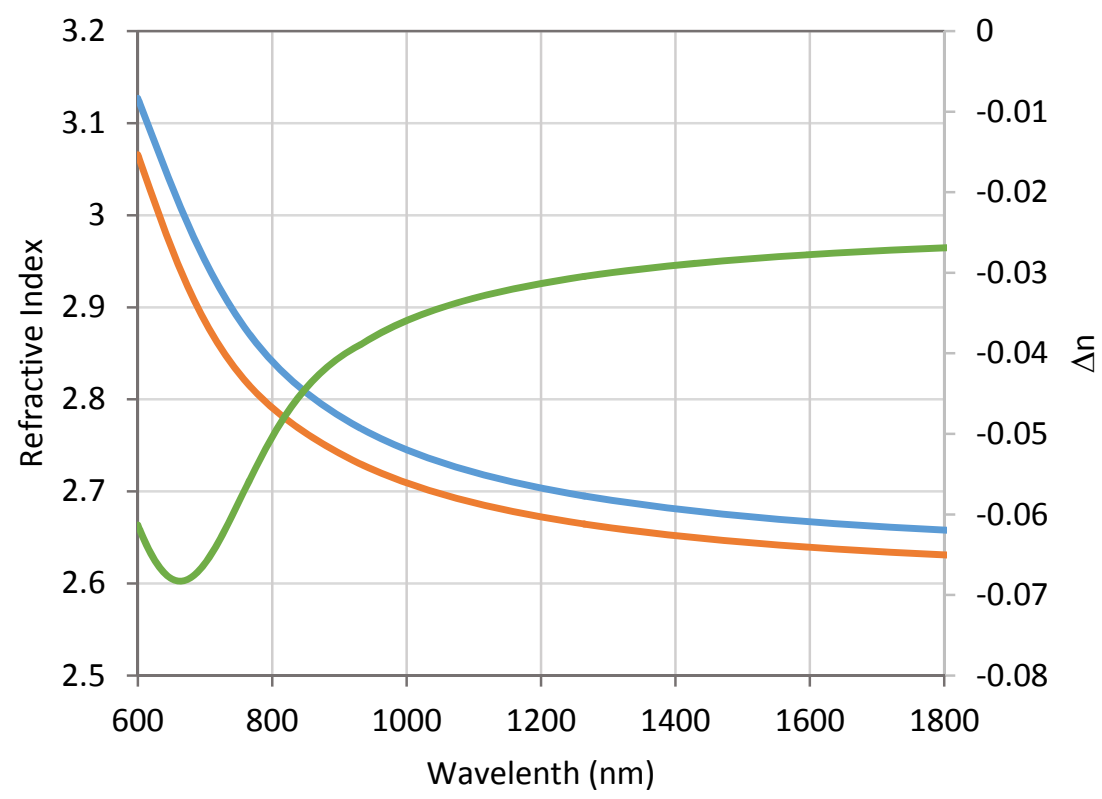

Figure 7: Refractive index dispersion curve before (blue) and after exposure (orange) at $808 \mathrm{~nm}$. Refractive index variation (green).

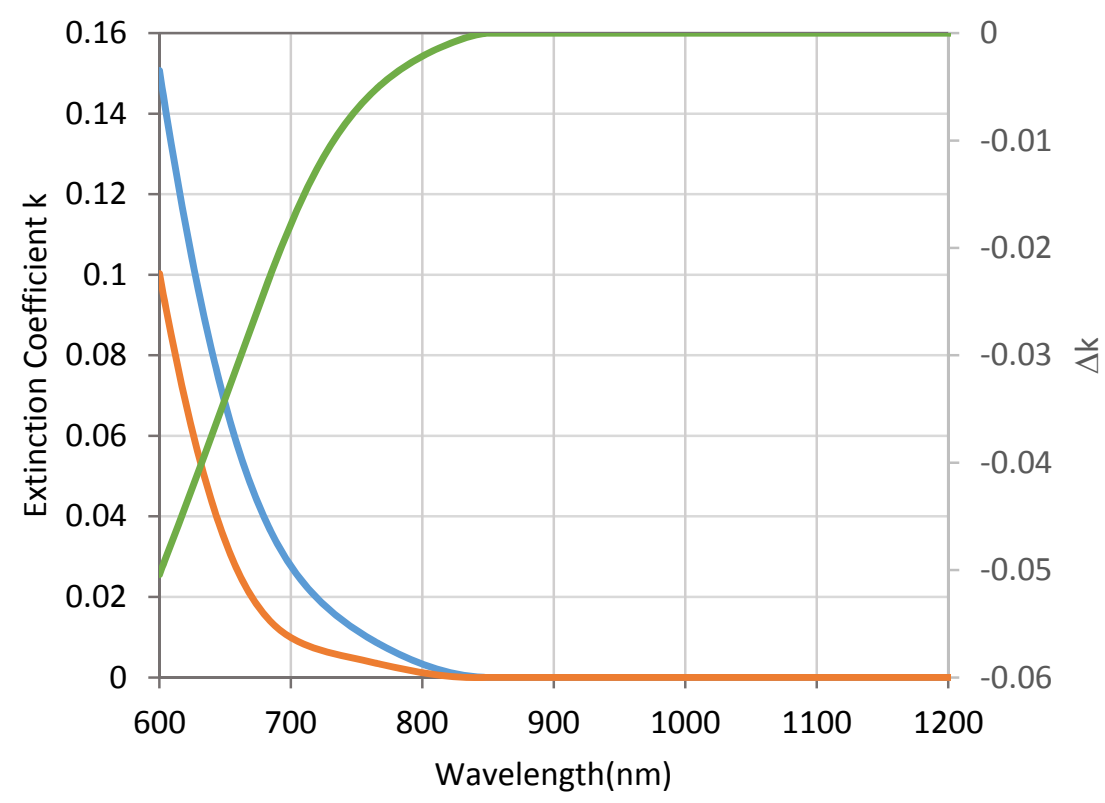


Figure 8: Extinction coefficient dispersion curve before (blue) and after exposure (orange) at 808 nm. Extinction coefficient variation (green).

Regarding the real part of the refractive index, it is decreasing after exposure. This decrease is quite large. An analysis using Tauc-Lorentz model reveals no photo-expansion or contraction of the film (extracted thickness varies from 548.1 to $548.0 \mathrm{~nm}$ and this change can be neglected). Such a negligible change of physical thickness (if it exist) was confirmed by surface profile measurements using a ZYGO Newview 7300 optical profilometer. Such a measurement did not reveal any measurable elevation change between the unexposed and exposed area, meaning that thickness was unaffected by the $808 \mathrm{~nm}$ exposure. Then we studied the kinetics of refractive index change on exposure dosage, for various dosages between 0 and saturation and modeled this change with a hyperbolic function [47] (Fig. 9). One can see that this model allow accurately predicting the refractive index change at any dosage with a precision better than $\pm 5 \%$. The saturation is reached after exposing the glass with dosage higher than $500 \mathrm{~kJ} / \mathrm{cm}^{2}$ at $808 \mathrm{~nm}$. Such a large dosage is required because of the low photosensitivity of the glass (slope at fluence $=0$ ) that is achieved at $808 \mathrm{~nm}$ because we wanted to secure a very low gradient of refractive index within the layer (less than $3 \% / \mu \mathrm{m}$ at $808 \mathrm{~nm}$ ). Faster refractive index change could be obtained by decreasing the exposure wavelength. 


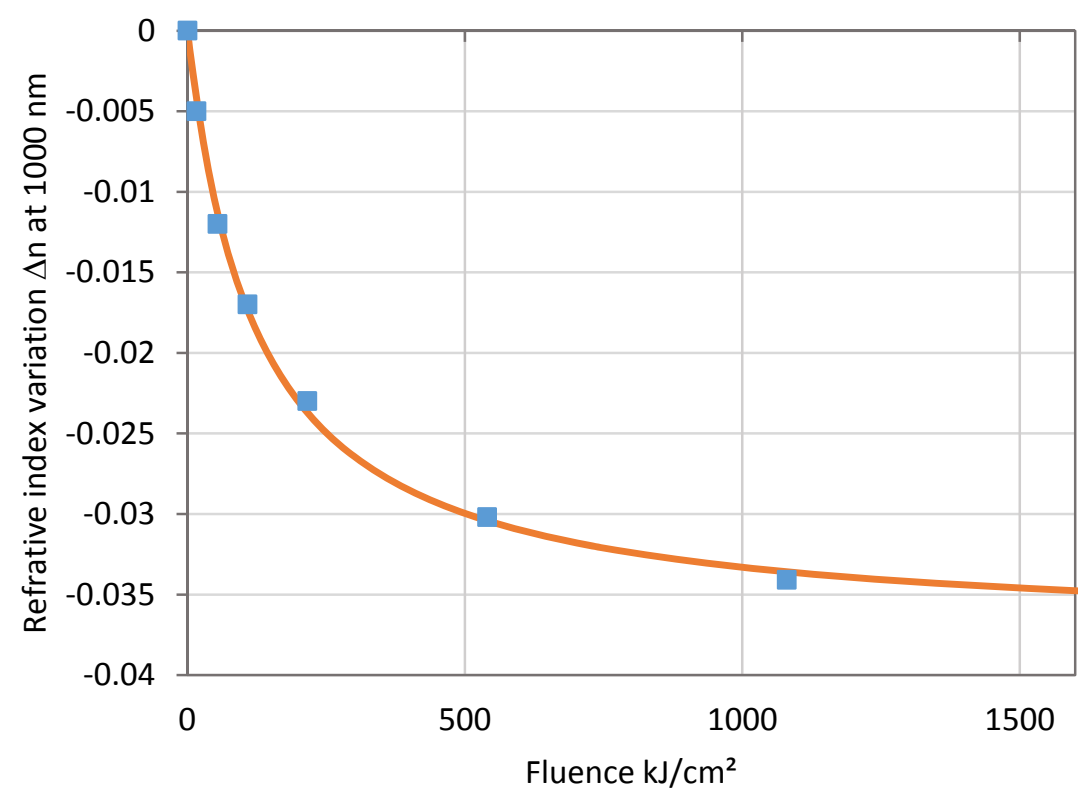

Figure 9: Photoinduced refractive index variation on fluence: in blue - measurement points, in orange -fit

Table 2. Parameters used for TL fitting of AMTIR-1 spectroscopic data measured before and after $808 \mathrm{~nm}$ exposure. Due to the level of noise of our measurements, the precision for each of the determined parameters is better than $1 \%$.

\begin{tabular}{ccccccccccc}
\hline & $\boldsymbol{E}$ & ETL & A1 & E01 & C1 & A2 & E02 & C2 & t & EF \\
\hline Unexposed & 1,82 & 1,46 & 87,48 & 3,92 & 3,82 & 3,96 & $-1,80$ & 0,71 & 548.1 & 0.0040 \\
Exposed & 1.78 & 1.48 & 83.7 & 4.12 & 3.84 & 5.08 & -1.82 & 0.66 & 548.0 & 0.0044 \\
\hline
\end{tabular}

\section{d. Glass stability in dark}

In order to be able to use these layer for optical application, it is mandatory to have stable layer with time. We thus studied the stability of the fabricated layers before and after exposure. Because of the photosensitivity of the layers, exposed and unexposed sample were stored in black box to avoid sunlight exposure and transmission was measured on those samples after different durations of storage: $0,10,20$ and 70 days. 
The transmission curves in Figure 10 illustrate the results obtained with unexposed sample. After being stored for 20 days in the dark, the transmission measured on the AMTIR-1 layers has slightly shifted towards the short wavelength range and an increase of the transmission can also be observed on the near-bandgap range. However, such an evolution could not be further noticed for longer storage duration. The transmission curves were then fitted with the TL model. A refractive index variation equal to $\Delta \mathrm{n}=-0.006$ was extracted. This decrease was associated with some natural relaxation and reconfiguration of the layers. It is worth mentioning that the previously shown results in section 4 were obtained taking into account this effect, the values of 0.04 at $1 \mu \mathrm{m}$ being the one expected after this drift has occurred. The post-exposition stability was tested on a sample issued from another sample that was exposed at $808 \mathrm{~nm}$ until refractive index saturation using the same procedure that was used with the unexposed sample (Figure 11). No significant differences can be detected. The transmissions spectra are identical proving that the photo-induced refractive index changes are permanent. 


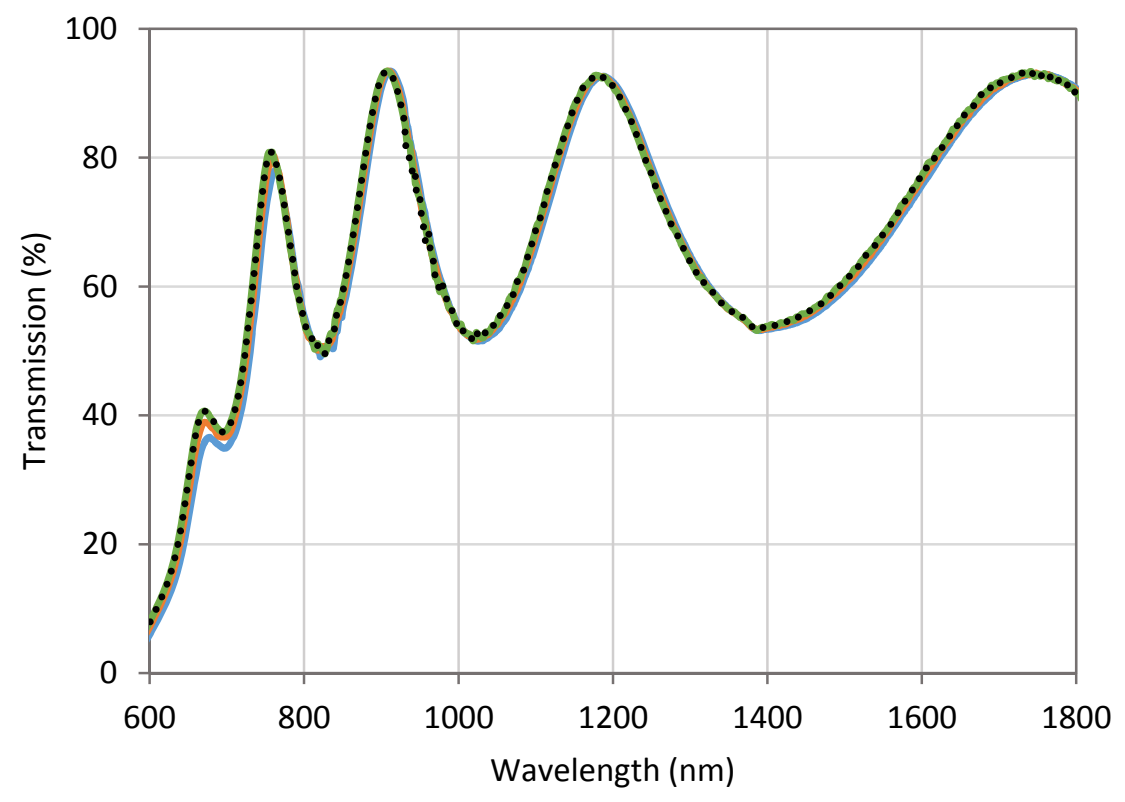

Figure 10: Spectral dependence of the transmission measured on an unexposed sample at different duration of storage in dark: in blue - 0 days, in orange - 10 days, in green - 20 days and black points - 70 days.

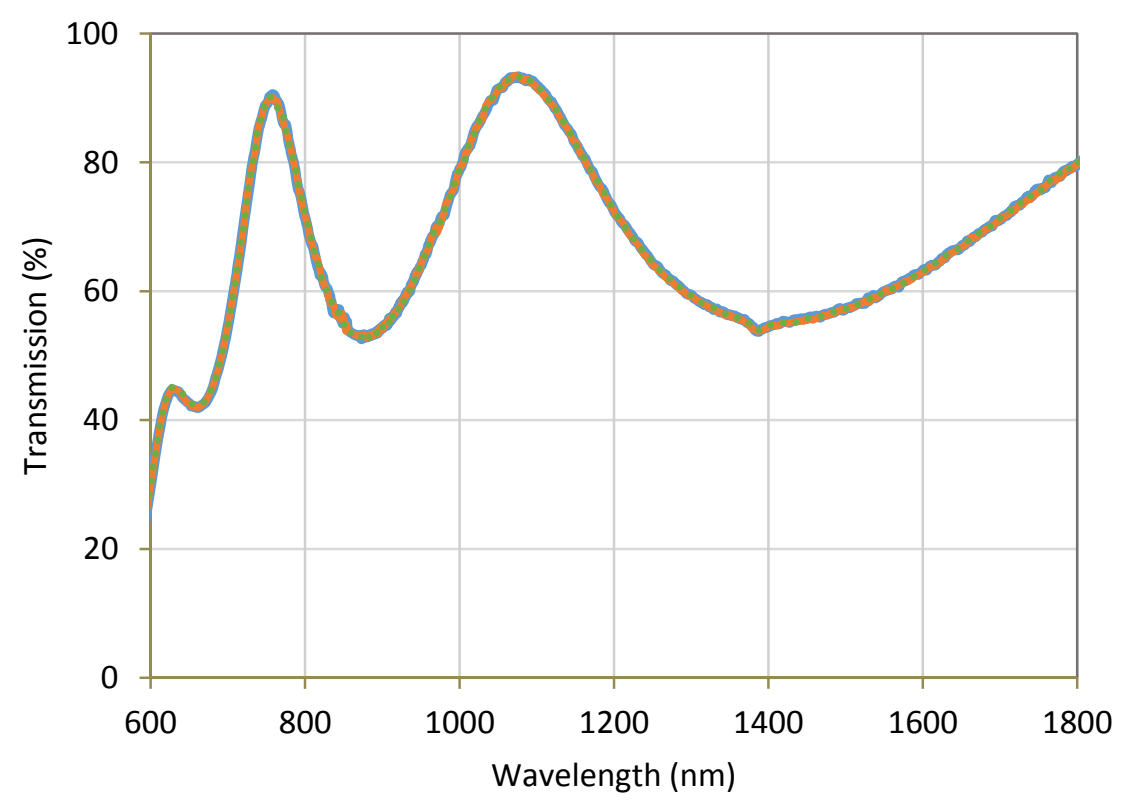

Figure 11: Spectral dependence of the transmission measured on an exposed sample at different duration of storage in dark: in blue - 0 days, in dots orange - 10 days and in green -20 days. 
These results prove that unexposed and exposed AMTIR-1 based layers are stable in time and can are therefore compatible with the fabrication of stable and durable optical elements. It is clear that such stability requires keeping these layers in dark because of their photosensitivity and outside application would require protecting these layers with additional layer that would reject all radiation around $800 \mathrm{~nm}$.

\section{Conclusion}

Using an electron beam deposition (EBD) process, AMTIR-1 layers were produced and characterized using reverse engineering by spectrophotometry with Tauc Lorent Model. This model was used to determine the optical constants (refractive index, extinction coefficient and bandgap) simultaneously in the regions of non-absorption and high absorption. In parallel a study of the chemical composition of EBD film shows that the compositions of thin films and the original bulk material are close.

Photosensitivity of AMTIR-1 layer was studied using the radiation form a laser diode with wavelength at $808 \mathrm{~nm}$. The transmission curve of the exposed layer is shifted towards shorter wavelength directly linked to a photo-bleaching effect. No variation of the layer thickness were measured. The photo-induced refractive index changes reached -0.05@800 nm.

Finally, a study of the stability of the unexposed and exposed layers shows that after a small drift of the layers properties during the first 20 days, the layers become stable and are therefore a good candidate for the fabrication of high quality optical elements.

\section{Bibliography}

[1] B. Mizaikoff, R. Göbel, R. Krska, K. Taga, R. Kellner, M. Tacke, A. Katzir, Infrared fiberoptical chemical sensors with reactive surface coatings, Sensors and Actuators B: Chemical 29, $58-63(1995)$ 
[2] S.R. Ovshinsky,'Reversible Electrical Switching Phenomena in Disorderes Structures", Physical Review Letters, vol 21, p. 1450, 1968.

[3] Wong, H.-S.P.; Raoux, S.; SangBum Kim; Jiale Liang; Reifenberg, John P.; Rajendran, B.; Asheghi, Mehdi; Goodson, Kenneth E., "Phase Change Memory," in Proceedings of the IEEE 98, 2201-2227 (2010)

[4] Kolobov, A. V. \& Tominaga, J. Chalcogenide glasses in optical recording: recent progress. J. Optoelectron. Adv. Mat. 4, 679-686 (2002).

[5] N. Mehta, application of chalcogenide glasses in electronics and optoelectronics: a review, Journal of Scientific \& Industral Research 65; 777-786 (2006)

[6] N. Singh, D. D. Hudson, R. Wang, E. C. Mägi, D.-Y. Choi, C. Grillet, B. Luther-Davies, S. Madden, and B. J. Eggleton, "Positive and negative phototunability of chalcogenide (AMTIR1) microdisk resonator," Opt. Express 23, 8681-8686 (2015)

[7] V. Nazabal, M. Cathelinaud, W. Shen, P. Nemec, F. Charpentier, H. Lhermite, M. Anne, J. Capoulade, F. Grasset, A. Moreac, S. Inoue, M. Frumar, J. Adam, M. Lequime, and C. Amra, "Chalcogenide coatings of Ge15Sb20S65 and Te20As30Se50," Appl. Opt. 47, C114-C123 (2008).

[8] Shimakawa, K., Kolobov, A. \& Elliott, S. R. Photoinduced effects and metastability in amorphous semiconductors and insulators. Adv. Phys. 44, 475-588 (1995).

[9] Fritzsche, H. Light induced structural changes in glasses, in insulating and semiconducting glasses (ed. Boolchand, P.) 653-690 (2000).

[10] T Uchino, Mechanism of Photoinduced Changes in the Structure and Optical Properties of Amorphous $\mathrm{As}_{2} \mathrm{~S}_{3}$, Phys. Rev. Lett. 85 (2000) 330

[11] H. Hamanaka, K. Tanaka, A. Matsuda, S. Iizima, Reversible photo-induced volume changes in evaporated As2S3 and As4Se5Ge1 films, Solid State Communications, Volume 19, Issue 6, July 1976, Pages 499-501 
[12] K. Tanaka, A. Saitoh, N. Terakado, “Giant photo-expansion in chalcogenide glass, Journal of optoelectronics and advanced. 8, December 2006, p. $2058-2065$

[13] S. Rajagopalan, K.S. Harshavardhan, L.K. Malhotra, K.L. Chopra, Photo-optical changes in Ge-chalcogenide films, Journal of Non-Crystalline Solids 50, 29-38 (1982)

[14] Kolobov, A. V. \& Tominaga, J., "Chalcogenide glasses in optical recording: recent progress," J. Optoelectron. Adv. Mat. 4, 679-686 (2002).

[15] A.V. Kolobov and S.R. Elliott, "Reversible photo-amorphization of crystalline films of As50Se 50", Journal of Non-Crystalline Solids 189 (1995) 297-300

[16] Pfeiffer, G., Paesler, M. A. \& Agarwal, S. C. "Reversible photodarkening of amorphous arsenic chalcogens”. J. Non-Cryst. Sol. 130, 111-143 (1991)

[17] S. Ducharne, J. Hautala, P.C. Taylor, "Photdarkening profiles and kinetics in chalcogenide glasses,” Phycical Review B 41, 12250-12260 (1990)

[18] G. Yang, H. Jain, A. Ganjoo, D. Zhao, Y. Xu, H. Zeng and G. Chen, "A photo-stable chalcogenide glass," Optics Express 16, 10565-10571, 2008

[19] W. Shen, M. Cathelinaud, M. Lequime, V. Nazabal et X. Liu, "Photosensitive post tuning of chalcogenide Te20As30Se50 narrow bandpass filters," Optics communications, vol. 281, pp. 3726-3731, 2008

[20] W. Shen, M. Cathelinaud, M. Lequime, F. Charpentier et V. Nazabal, "Light trimming of a narrow bandpass filter based on a photosensitive chalcogenide spacer," Optics express, vol. 16, pp. 373-383, 2008

[21] D. A. Turnbull, J. S. Sanghera, V. Nguyen, and I. D. Aggarwal, "Fabrication of waveguides in sputtered films of GeAsSe glass via photodarkening with above bandgap light," Mater. Lett. $58,51-54(2004)$. 
[22] A. Saliminia, A. Villeneuve, T. V. Galstyan, S. LaRochelle, and K. Richardson, "Firstand second-order Bragg gratings in single-mode planar waveguides of chalcogenide glasses," J. Lightwave Technol. 17, 837-842 (1999).

[23] N. J. Baker, H. W. Lee, I. C. M. Littler, C. M. de Sterke, B. J. Eggleton, D. Y. Choi, S. Madden, and B. Luther-Davies, "Sampled Bragg gratings in chalcogenide (As2S3) ribwaveguides," Opt. Express 14, 9451-9459 (2006).

[24] S. Ramachandran and S. G. Bishop, "Photoinduced integratedoptic devices in rapid thermally annealed chalcogenide glasses,” IEEE J. Sel. Top. Quantum Electron. 11, 260-270 (2005).

[25] P. Němec, S. Zhang, V. Nazabal, K. Fedus, G. Boudebs, A. Moreac, M. Cathelinaud, and X.-H. Zhang, Photo-stability of pulsed laser deposited GexAsySe100-x-y amorphous thin films, Opt. Express, 18 [22] 22944-22957 (2010)

[26] R.P. Wang, A.V. Rode, S.J. Madden, C.J. Zha, R.A. Jarvis, B. Luther-Davies, "Structural relaxation and optical properties in amorphous Ge33As12Se55 films," Journal of NonCrystalline Solids 353, 950-952 (2007)

[27] D.-Y. Choi, S. Madden, A. Rode, R. Wang, B. Luther-Davies, "Fabrication of low loss Ge33As12Se55 (AMTIR-1) planar waveguides,” Applied Physics Letters, 91, 011115 (2007) [28] R.A. Jarvis, R.P. Wang, A.V. Rode, C. Zha, B. Luther-Davies, “Thin film deposition of Ge33As12Se55 by pulsed laser deposition and thermal evaporation: Comparison of properties," Journal of Non-Crystalline Solids 353, Pages 947-949 (2007)

[29] Xueqiong Su, Rongping Wang, Barry Luther-Davies, Li Wang, The dependence of photosensitivity on composition for thin films of GexAsySe1-x-y chalcogenide glasses, Appl. Phys. A-Mater. Sci. Process. 113 [3] 575-581 (2013), 
[30] Guang Yang, Himanshu Jain, Ashtosh Ganjoo, Donghui Zhao, Yinsheng Xu, Huidan Zeng, Guorong Chen, A photo-stable chalcogenide glass, Opt. Express 16 [14] 10565-10571 (2008),

[31] F. Charpentier, M. Dussauze, M. Cathelinaud, G. Delaizir, E. Kamitsos, J.-L. Adam, B. Bureau et V. Nazabal, «Aging process of photosensitive chalcogenide films deposited by electron beam deposition,» Journal of Alloys and Compounds, vol. 509, pp. 7330-7336, 2011.

[32] R.Swanepoel, "Determination of the thickness and optical constants of amorphous silicon,” J. Phps. E: Sci. Instrum, Vol. 16 (1983)

[33] E Langereis, E Langereis, S B S Heil, H C M Knoops, W Keuning, M C M van de Sanden and W M M Kessels, "In situ spectroscopic ellipsometry as a versatile tool for studying atomic layer deposition,” J. Phys. D: Appl. Phys. 42 (2009) 073001

[34] G.E. Jellison Jr., F.A. Modine, "Parameterization of the optical functions of amorphous materials in the interband region,” Appl. Phys. Lett. 69 (1996), pp. 371-373 (Erratum: "Parameterization of the optical functions of amorphous materials in the interband region" [Appl. Phys. Lett. 69, 371 (1996)], idid. 69 (1996) 2137)

[35] G.E. Jellison Jr., V.I. Merkulov, A.A. Puretzky, D.B. Geohegan, G. Eresa, D.H. Lowndes, J.B. Caughman, "Characterization of thin-film amorphous semiconductors using spectroscopic ellipsometry," Thin Solid Films 377-378 (2000), pp. 68-73

[36] G.E. Jellison Jr., "Physics of optical metrology of silicon-based semiconductor devices A.C. Diebold (Ed.), Handbook of Silicon Semiconductor Metrology," Marcel Dekker, Inc., New York NY (2001), pp. 723-760

[37] B. von Blanckenhagen, D. Tonova, and J. Ullmann, “Application of the Tauc-Lorentz formulation to the interband absorption of optical coating materials," Appl. Opt. 41, 3137-3141 (2002) 
[38] D.V. Likhachev, N. Malkova, L. Poslavsky, "Modified Tauc-Lorentz dispersion model leading to a more accurate representation of absorption features below the bandgap," Thin Solid Films 589, 844-851, (2015)

[39] L. Gao, F. Lemarchand, M. Lequime, "Comparison of different dispersion models for single layer optical thin film index determination," Thin Solid Films 520, 501-509 (2011)

[40] L. Gao, F. Lemarchand, M. Lequime, "Reverse engineering from spectrophotometric measurements: performances and efficiency of different optimization algorithms," Appl. Phys. A $108,877-889(2012)$

[41] J. He, R. Xu, W. Li, K.-C. Qi, Y.-D. Jiang, "Dispersion model for optical constants of aSi:H,” Physica B 431, 120-126 (2013)

[42] R. Kronig, “On the theory of dispersion of X-rays,” J. Opt. Soc. Am. 12, 547-556 (1926) [43] Y.J. Cho, N.V. Nguyen, C.A. Richter, J.R. Ehrstein, B.H. Lee, J.C. Lee, "Spectroscopic ellipsometry characterization of high- $\kappa$ dielectric $\mathrm{HfO} 2$ thin films and the high-temperature annealing effects on their optical properties,” Appl. Phys. Lett., 80, 1249-1251 (2002) [44] X.-Y. Gao, H.-L. Feng, J.-M. Ma, Z.-Y. Zhang, J.-X. Lu, Y.-S. Chen, S.-E. Yang, J.-H. $\mathrm{Gu}$, "Analysis of the dielectric constants of the $\mathrm{Ag}_{2} \mathrm{O}$ film by spectroscopic ellipsometry and single-oscillator mode," Physica B 405 1922-1926 (2010)

[45] Julien Lumeau and Leonid B. Glebov, "Gradient of refractive index (GRIN) effect in photo-thermo-refractive glass,” Appl. Opt. 54, 1587-1593 (2015)

[46] Alexandre Joërg and Julien Lumeau; "Fabrication of binary volumetric diffractive optical elements in photosensitive chalcogenide AMTIR-1 layers," Optics Letters 40, 3233-3236 (2015)

[47] J. Lumeau and L. B. Glebov, "Modeling of the induced refractive index kinetics in photothermo-refractive glass," Optical Materials Express 3(1), 95-104 (2013) 
[48] A. Kovalskiy, A. C. Miller, H. Jain, and M. Mitkova, "In-situ measurements of x-ray induced silver diffusion into Ge30Se70 thin film,” J. Am. Ceram. Soc. 91, 760-765 (2008). 